\title{
Doubly Stochastic Coherence via Noise-Induced Symmetry in Bistable Neural Models
}

\author{
A. Zaikin, ${ }^{1}$ J. García-Ojalvo, ${ }^{2}$ R. Báscones,${ }^{2}$ E. Ullner, ${ }^{1}$ and J. Kurths ${ }^{1}$ \\ ${ }^{1}$ Institut für Physik, Potsdam Universität, Am Neuen Palais 10, D-14469 Potsdam, Germany \\ ${ }^{2}$ Departament de Física i Enginyeria Nuclear, Universitat Politècnica de Catalunya, Colom 11, E-08222 Terrassa, Spain
}

(Received 13 July 2002; published 23 January 2003)

\begin{abstract}
The generation of coherent dynamics due to noise in an activator-inhibitor system describing bistable neural dynamics is investigated. We show that coherence can be induced in deterministically asymmetric regimes via symmetry restoration by multiplicative noise, together with the action of additive noise which induces jumps between the two stable steady states. The phenomenon is thus doubly stochastic, because both noise sources are necessary. This effect can be understood analytically in the frame of a small-noise expansion and is confirmed experimentally in a nonlinear electronic circuit. Finally, we show that spatial coupling enhances this coherent behavior in a form of system-size coherence resonance.
\end{abstract}

DOI: 10.1103/PhysRevLett.90.030601

Rhythm generation is a long-standing problem in science, particularly in biological and cognitive science contexts $[1,2]$. A paradigm of this kind of self-sustained oscillating behavior in nonlinear systems is offered by limit cycles. But even in the absence of limit cycles, internal rhythms can be generated in nonlinear systems by the effect of noise. An early realization of this phenomenon was reported in a two-dimensional autonomous system when operating close to a limit cycle and was interpreted as a manifestation of stochastic resonance in the absence of external forcing [3]. An optimal amount of noise was also seen to lead to a maximally coherent output in an excitable system [4]. This effect, called coherence resonance, was studied in the well-known FitzHugh-Nagumo model, which has been extensively used to describe the dynamics of neural systems [5]. Coherence resonance has been confirmed in several experimental situations, such as in laser systems [6]. Furthermore, it has also been predicted in a system with two chaotic attractors [7] and in excitable media coupled via an inhibitor concentration, provided the coupled elements behave in antiphase [8].

A complete understanding of these different mechanisms of coherence resonance is very important for the study of rhythm generation in biological systems [2,9] and, in particular, in neural tissue. On the other hand, increasing experimental evidence has established in recent years that certain types of neurons frequently operate in a bistable regime [10]. Thus, the question arises whether noise can excite an autonomous coherent output in bistable neural systems. In this direction, both standard stochastic and coherence resonance have been observed in a symmetrically bistable FitzHugh-Nagumo model [11]. In the present Letter, we show that coherence can also be generated in the general asymmetric case, where the stability of the two stable steady states is not necessarily the same. We demonstrate that the mechanism of coherence enhancement in this situation is utterly different from the standard one, being based on the restoration of
PACS numbers: 05.40.-a, 05.70.Fh, 87.10.+e

symmetry induced by a multiplicative source of noise. This effect vividly contrasts with standard noise-induced phase transitions, where noise usually leads to the breaking of symmetry [12].

Doubly stochastic coherence (DSC) can be observed in an asymmetric system under the joint action of multiplicative and additive noises. Once multiplicative noise induces a symmetric bistable state in the system, due to the presence of optimal additive noise, coherence can be maximized in the output. Hence, the resulting coherence is doubly stochastic, since simultaneous optimization of two noise intensities is required in order to observe the phenomenon. The concept of doubly stochastic effects has been introduced recently as a new mechanism of noiseinduced phenomena in the context of harmonically driven systems [13]. These effects are usually possible due to the interplay between additive and multiplicative noise. In [13], multiplicative noise (in combination with spatial coupling) induces bistability in a simple monostable extended system, and additive noise induces synchronization with the external signal in that noiseinduced bistable regime. Such doubly stochastic resonance has been reported in simple electronic circuit models [14]. Following those lines, we have shown recently that doubly stochastic effects lead also to signal propagation in simple monostable media [15]. The synthesis of noise-induced transitions and noise-induced transport reported in [16] is also related to this kind of effects. In this Letter, we report the occurrence of DSC in a modified version of the well-known FitzHugh-Nagumo (FHN) model. The mechanism is explained theoretically in the framework of a small-noise expansion of the model, which extracts the systematic contribution of the multiplicative noise that accounts for the symmetry restoration. The results of this analysis and numerical results are confirmed by experiments on an electronic circuit. Finally, we show that this effect can be generalized for the case of spatially extended systems, where it leads to synchronization induced by multiplicative noise. 
We consider the following version of the FHN model:

$$
\begin{aligned}
& \varepsilon \frac{d u}{d t}=u(1-u)(u-a)-v, \\
& \frac{d v}{d t}=b u-v-u v \xi(t)+\zeta(t) .
\end{aligned}
$$

In a neural context, $u(t)$ represents the membrane potential of the neuron and $v(t)$ is related to the time-dependent conductance of the potassium channels in the membrane [5]. The dynamics of $u$ is much faster than that of $v$, as indicated by the small time-scale-ratio parameter $\varepsilon$. There are two mutually uncorrelated noise sources, represented by the $\delta$-correlated Gaussian noises $\xi(t)$ and $\zeta(t)$, with zero mean and correlations $\left\langle\xi(t) \xi\left(t^{\prime}\right)\right\rangle=\sigma_{m}^{2} \delta\left(t-t^{\prime}\right)$ and $\left\langle\zeta(t) \zeta\left(t^{\prime}\right)\right\rangle=\sigma_{a}^{2} \delta\left(t-t^{\prime}\right)$. The additive noise is inserted in the slow-variable equation, as in most studies of coherence resonance [4]. The multiplicative noise $\zeta(t)$ is interpreted in the Stratonovich sense [12].

In what follows we use the parameters $a=0.15, b=$ 0.12 , and $\varepsilon=0.01$, for which the deterministic system has two stable fixed points with different stability (i.e., with different thresholds of escape through the extrema of the $u$ nullcline), as shown in Fig. 1(a) (curve 1 and its crossing points with the $u$ nullcline). Additive noise induces here jumps between these two states, but the escape times are very different in the two states. This behavior is shown in Fig. 1(b), as obtained from numerical simulations of model (1) for the above-mentioned parameters.

The effect of multiplicative noise in this system can be determined by analyzing the systematic effect it produces in the system dynamics due to the fact that the corresponding fluctuating term in the $v$ equation has a nonzero average value. Computation of this average value by means of standard techniques [17] leads to the following effective deterministic model, which can be considered as a first order approximation in a small-noise expansion of $\xi(t)[12]$ :
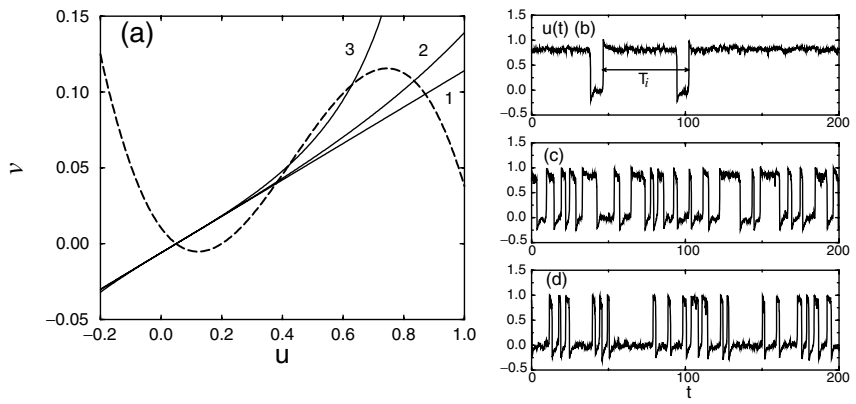

FIG. 1. (a) Nullcline plot of the FHN model (1). Dashed line: $u$ nullcline $(\dot{u}=0)$; solid lines: $v$ nullclines $(\dot{v}=0)$ for three different values of the multiplicative noise intensity: $\sigma_{m}^{2}=0.0$ (curve 1), 0.2 (curve 2), and 2.0 (curve 3). (b)-(d) Time evolution of the activator variable $u$ for the previous three multiplicative noise intensities: (b) $\sigma_{m}^{2}=0.0$, (c) 0.2 , (d) 2.0 . The intensity of additive noise is fixed to $\sigma_{a}^{2}=2 \times 10^{-4}$; other parameters are given in the text.

$$
\begin{gathered}
\varepsilon \frac{\partial u}{\partial t}=u(1-u)(u-a)-v, \\
\frac{\partial v}{\partial t}=b u-v+\frac{\sigma_{m}^{2}}{2} u^{2} v+\zeta(t) .
\end{gathered}
$$

The nullclines of this model for two nonzero values of $\sigma_{m}^{2}$ are shown in Fig. 1(a), as curves 2 and 3. It can be seen that for an intermediate value of $\sigma_{m}^{2}$, corresponding to curve 2 , the two states are equally stable and the escape times are basically identical. As a result, jumps in the output of the system are more equidistant [Fig. 1(c)]. For larger multiplicative noise intensity the asymmetry increases again, this time reversed, as shown in curve 3 of Fig. 1(a), and the system spends more time in the lower state, as shown in Fig. 1(d) (in fact, in this extreme case the upper steady state has turned unstable, and the system becomes excitable).

Hence, an optimal amount of multiplicative noise optimizes the symmetric response of the system. In that situation, we can expect additive noise to be more effective in producing coherence, since the potential barrier heights (and thus the corresponding escape times) are the same in the two jump directions. To quantify this expected coherence enhancement, we have measured the normalized variance of subsequent periods $T_{i}$. The illustration of the definition of $T_{i}$ is depicted in Fig. 1(b). The normalized variance, which is called the coherence parameter [4], is determined as $R=\sqrt{\sigma_{T}^{2}} /\left\langle T_{i}\right\rangle$, where $\sigma_{T}^{2}$ is the variance of the sequence $T_{i}$, and $\left\langle T_{i}\right\rangle$ is its average value. The dependence of $R$ on the multiplicative noise intensity for the time series depicted in Figs. 1(b)-1(d) is shown in Fig. 2 (left). It is clearly seen that $R$ first decreases to some minimum value and then increases again. The minimal $R$ corresponds to the highest degree of periodicity in the system output and is a manifestation of stochastically induced coherence. A similar behavior occurs for varying the strength of the additive noise as well, as shown in the inset of Fig. 2 (left). Different values of the excitation threshold correspond to different optimal intensities of the noise. To optimize the periodicity, one should vary both the threshold (provided by
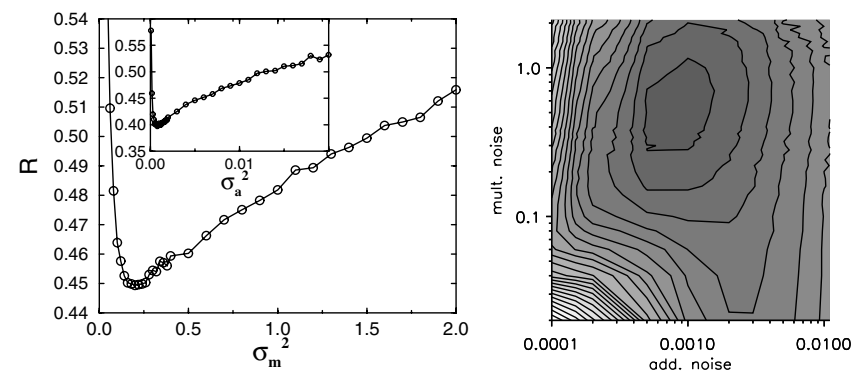

FIG. 2. Left: coherence parameter $R$ vs intensity of the multiplicative and additive (inset plot) noises. $\sigma_{a}^{2}=2 \times 10^{-4}$ and $\sigma_{m}^{2}=0.5$, respectively. Right: contour plot of the coherence parameter $R$ vs intensity of the multiplicative and additive noises (darker gray corresponds to smaller values of $R$ ). 


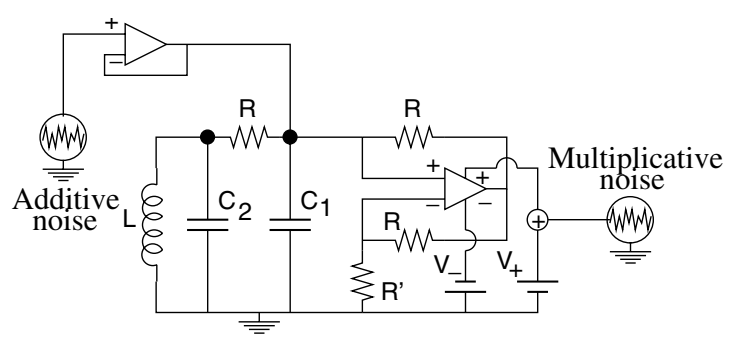

FIG. 3. Nonlinear electronic circuit with two asymmetrically stable steady states. The values of the elements are $R=270 \Omega$, $L=10 \mathrm{mH}, C_{1}=1 \mathrm{nF}, C_{2}=10 \mathrm{nF}, R^{\prime}=220 \Omega, V_{-}=5 \mathrm{~V}$, and $V_{+}=2 \mathrm{~V}$. The operational amplifier is taken from a TL082 integrated circuit.

multiplicative noise) and the intensity of additive noise. Both noise intensities need to be tuned in order to optimize periodicity in the output [see Fig. 2 (right)], and hence we call this effect doubly stochastic coherence.

With the aim of confirming experimentally the phenomenon of DSC via noise-induced symmetry, we have designed a circuit (Fig. 3), which has two asymmetrically stable steady states. In this circuit, the difference between the positive and negative voltages feeding the operational amplifier provides the asymmetry in the stability of the two fixed points. Multiplicative noise acts on the positive voltage $V_{+}$, which is a parameter that changes the stability of the higher voltage fixed point of the circuit [18]. A second source of noise, which acts as a signal, induces jumps between the two stable states and acts as an additive noise. The noise is produced electronically by amplifying shot noise from a junction diode [19].

Following the numerical approach, we fix the intensity of additive noise and increase that of multiplicative noise. First, the upper steady state is more stable than the lower one, and the system spends more time in the former [Fig. 4(a)]. As the strength of multiplicative noise increases, the situation is reversed [Fig. 4(c)], passing through a symmetric regime for intermediate noise [Fig. 4(b)]. Calculating the coherence parameter $R$ for the experimental time traces, we find clearly that multiplicative noise enhances coherence via the appearance of symmetry [Fig. 4(d)].

We have also examined the effect of spatial coupling on a set of distributed bistable FHN oscillators subject to two noise sources. The model is now given by

$$
\begin{gathered}
\varepsilon \frac{\partial u_{i}}{\partial t}=u_{i}\left(1-u_{i}\right)\left(u_{i}-a\right)-v_{i} \\
+\frac{D}{2}\left(u_{i+1}+u_{i-1}-2 u_{i}\right), \\
\frac{\partial v_{i}}{\partial t}=b u_{i}-v_{i}-u_{i} v_{i} \xi_{i}(t)+\zeta_{i}(t),
\end{gathered}
$$

where $D$ denotes the strength of coupling and the noise terms are now $\delta$ correlated also in space, with $\left\langle\xi_{i}(t) \xi_{j}\left(t^{\prime}\right)\right\rangle=\sigma_{m}^{2} \delta\left(t-t^{\prime}\right) \delta_{i j}$ and $\left\langle\zeta_{i}(t) \zeta_{j}\left(t^{\prime}\right)\right\rangle=$ $\sigma_{a}^{2} \delta\left(t-t^{\prime}\right) \delta_{i j}$.

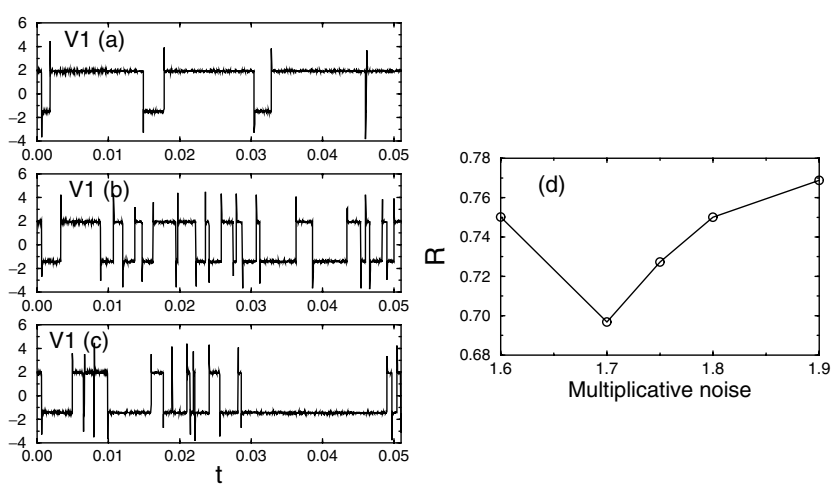

FIG. 4. Time evolution of the voltage drop $V_{1}$ through capacitor $C_{1}$ for the circuit represented in Fig. 3, for three different intensities of the multiplicative noise (measured as peak-to-peak amplitude of the random voltage): (a) $1.6 \mathrm{~V}$, (b) $1.7 \mathrm{~V}$, and (c) $1.9 \mathrm{~V}$. Additive noise intensity is fixed to 0.88 V. (d) Coherence parameter vs multiplicative noise intensity.

We now study the joint effect of additive and multiplicative noise on the spatiotemporal evolution of this extended system, using a binary coding for the activator variable $u_{i}(t)$, associating black or white to each one of the two fixed points of the local bistable dynamics. The numerical simulation results are shown in Fig. 5 for three values of $\sigma_{m}^{2}$ and a fixed $\sigma_{a}^{2}$. As expected, the local dynamics becomes more regular for an optimal amount of multiplicative noise, as happens with an isolated FHN element. However, remarkably enough, the most temporally coherent case corresponds also to the most spatially uniform behavior of the system as a whole. To characterize such a synchronized coherence, we calculate the coherence parameter $R$ for the mean field $m(t)=\sum_{i} u_{i}$. The dependence of this parameter on the intensity of multiplicative noise is shown in Fig. 6(a) for a system of 50 coupled elements. The dependence is nonmonotonic, reflecting the DSC characteristic of isolated elements,
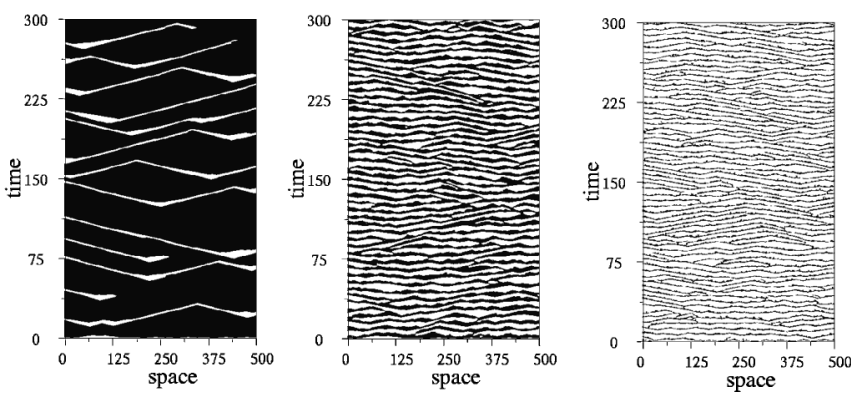

FIG. 5. Spatiotemporal evolution of a chain of FHN oscillators in the bistable regime for three intensities of the multiplicative noise. From left to right, $\sigma_{m}^{2}=0.01,0.2,4$. Additive noise is fixed to $\sigma_{a}^{2}=4 \times 10^{-4}$. Coding is binary, with black corresponding to the upper fixed point and white to the lower one. Other parameters are $D=30, a=0.15, b=0.12$, and $\varepsilon=0.01$.

030601-3 

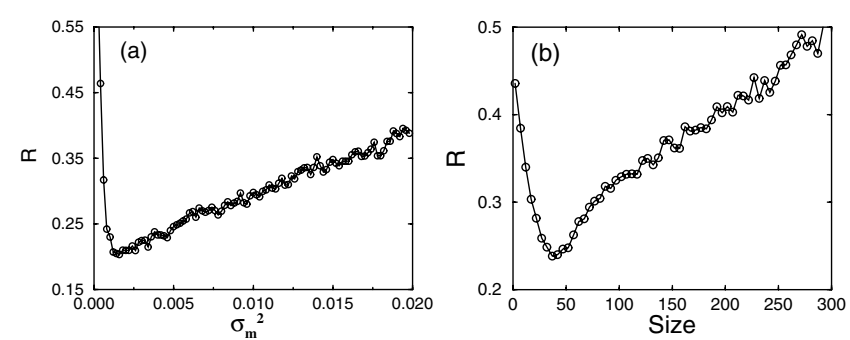

FIG. 6. (a) Coherence parameter $R$ of the mean field $m(t)$ vs intensity of the multiplicative noise for a system with 50 coupled elements. (b) The dependence of $R$ on the size of the system $\left(\sigma_{m}^{2}=0.005\right)$.

although in this case the parameter measures also the degree of synchronization in the system. Furthermore, Fig. 6(b) shows that increasing the number of elements in the ensemble first increases the coherence of the output $(R$ initially decreases), due to the synchronization of the elements, but further increase of the system size leads to a loss of synchronization, and thus $R$ increases again. The result is a system-size coherence resonance (cf. with system-size stochastic resonance, which happens in externally forced systems [20]). In a neural context, this property could imply that neurons benefit from coupling in networks of optimal size for the organization of a pacemaker.

In conclusion, we have shown that bistable models of neural dynamics exhibit doubly stochastic coherence via noise-induced symmetry. This mechanism of rhythm generation arises whenever the two stable steady states of the system have different escape thresholds. An optimal amount of multiplicative noise renders the two fixed points equally stable, and tuning the additive noise in this noise-induced symmetric situation maximizes the coherent behavior in the system. The influence of multiplicative noise can be explained in terms of an effective model that contains the systematic effect of the noise term. These results have been confirmed by experimental measurements on a bistable nonlinear electronic circuit. From a second standpoint, we have shown that this effect leads to synchronized behavior in spatially distributed systems. In this case, this coherence enhancement also exhibits a resonance with respect to the size of the system; i.e., there is some optimal size of the system for which the output is the most periodic one. Our study has been performed in the general framework of the paradigmatic FHN model, in a bistable asymmetric regime which is realistic for biological systems [10], and hence we expect that our findings could be of importance for understanding the mechanisms of periodicity generation in neural and other excitable media.
We thank H. Busch for technical help. A. Z. acknowledges support from CESCA-CEPBA through the EC IHP Program (HPRI-1999-CT-00071) and from ESA (MPA AO-99-030), J. G. O. from DGES (Spain, BFM2001-2159 and BFM2002-04369), E. U. from the International MP Research School on Biomimetic systems, and J. K. from SFB 555 (Germany).

[1] A.T. Winfree, The Geometry of Biological Time (Springer, Berlin, 1980).

[2] R. Engbert, C. Scheffczyk, R. T. Krampe, M. Rosenblum, J. Kurths, and R. Kliegl, Phys. Rev. E 56, 5823 (1997).

[3] G. Hu, T. Ditzinger, C. Z. Ning, and H. Haken, Phys. Rev. Lett. 71, 807 (1993).

[4] A. Pikovksy and J. Kurths, Phys. Rev. Lett. 78, 775 (1997).

[5] J. Keener and J. Snyder, Mathematical Physiology (Springer, New York, 1998).

[6] G. Giacomelli, M. Giudici, S. Balle, and J. R. Tredicce, Phys. Rev. Lett. 84, 3298 (2000); J. M. Buldú, J. GarcíaOjalvo, C. R. Mirasso, M. C. Torrent, and J. M. Sancho, Phys. Rev. E 64, 051109 (2001).

[7] C. Palenzuela, R. Toral, C. Mirasso, O. Calvo, and J. Gunton, Europhys. Lett. 56, 347 (2001).

[8] E. Volkov, M. Stolyarov, A. Zaikin, and J. Kurths (to be published).

[9] J. Collins and I. Stewart, J. Nonlinear Sci. 3, 349 (1993).

[10] S.W. Hughes, D.W. Cope, T. I. Tóth, S. R. Williams, and V. Crunelli, J. Physiol. (London) 517, 805 (1999).

[11] B. Lindner and L. Schimansky-Geier, Phys. Rev. E 61, 6103 (2000); F. Moss, J. K. Douglass, L. Wilkens, D. Pierson, and E. Pantazelou, Ann. N.Y. Acad. Sci. 706, 26 (1993).

[12] J. García-Ojalvo and J. M. Sancho, Noise in Spatially Extended Systems (Springer, New York, 1999).

[13] A. Zaikin, J. Kurths, and L. Schimansky-Geier, Phys. Rev. Lett. 85, 227 (2000).

[14] A. Zaikin, K. Murali, and J. Kurths, Phys. Rev. E 63, 020103(R) (2001).

[15] A. Zaikin, J. García-Ojalvo, L. Schimansky-Geier, and J. Kurths, Phys. Rev. Lett. 88, 010601 (2002).

[16] P. Reimann, R. Kawai, C. Van den Broeck, and P. Hänggi, Europhys. Lett. 45, 545 (1999).

[17] J. M. Sancho and J. García-Ojalvo, in Stochastic Processes in Physics, Chemistry, and Biology, edited by J. Freund and T. Pöschel (Springer, New York, 2000), pp. 235-246.

[18] R. Báscones, J. García-Ojalvo, and J. M. Sancho, Phys. Rev. E 65, 061108 (2002).

[19] M. Löcher, D. Cigna, and E. R. Hunt, Phys. Rev. Lett. 80, 5212 (1998).

[20] A. Pikovksy, A. Zaikin, and M. A. de la Casa, Phys. Rev. Lett. 88, 050601 (2002). 